\title{
Vî̀ GIẢI THUẬT SO SÁNH HAI TÙ TRONG THUẬT TOÁN TRUY NGUYÊN ĐỒNG NHẤT
}

\author{
PHƯONG MINH NAM ${ }^{(1)}$, LÊ TIÊN VƯONG ${ }^{(2)}$
}

\begin{abstract}
The problems for automatical indentification algorithm in databases with Latinized names has discussed overview in [2], [3], this paper is issuing detail algorithms that compares words of Latinized name following occurrences probability.
\end{abstract}

Trong các hệ thống thông tin không đầy đủ, việc tìm kiếm, đồng nhất một đối tượng dựa trên một số đặc trưng nào đó luôn là một vấn đề được nhiều nhà nghiên cứu quan tâm và có nhiều hướng giải quyết khác nhau. Các phần mềm cơ sở dữ liệu (CSDL) thương phẩm như SQL Server, Oracle, Sysbase... đều cài đặt các toán tứ tìm kiếm thông minh như LIKE, MATCH, SOUND... Một trong các cách tiếp cận được tập trung nghiên cứu là sử dụng lý thuyết tập mờ để biểu diễn và tìm kiểm thông tin khá thành công.

Trong [3] đã đưa ra một cách tiếp cận tìm kiếm mờ dựa trên cơ sở lý thuyết thống kê chọn mẫu và lập ma trận sai sót (ma trận độ đo của sai lệch). Việc đồng nhất hai đối tượng bất kỳ trong một CSDL có thể phải sử dụng tới một số thuộc tính nào đó. Không làm mất tính tồng quát, ở đây chỉ đưa ra cách thức đồng nhất dựa trên việc so sánh hai xâu kí tự biểu diễn họ và tên của đối tượng.

\section{1. ĐẶT BÀI TOÁN}

Giả sử rằng thuộc tính họ và tên có độ dài trung bình không quá 24 kí tự.

$X, Y$ là hai giá trị của thuộc tính này với $X=\left(X_{1} X_{2} \ldots X_{n}\right)$ và $Y=\left(Y_{1} Y_{2} \ldots Y_{n}\right)$, trong đó $X_{i}, Y_{j}, i, j<24$ là kí hiệu cho từng kí tự thuộc xâu $X$ và $Y$. Gọi $\rho(X, Y)$ là hàm độ đo giữa hai xâu $X$ và $Y ; \delta$ là một số cho trước. Khi đó nếu $\rho(X, Y)<\delta$ nói rằng $X$ và $Y$ là cùng một lớp theo ngưỡng $\delta$.

Vấn đề cần giải quyết là :

- Xác định ngưỡng độ đo $\delta$.

- Xác định thuật toán chấp nhận được để so sánh hai sâu (hai giá trị họ và tên ) theo ngưỡng độ đo đã cho.

Trong bài này chúng tôi quan sát và giải quyết trong trường hợp các giá trị thuộc tính họ và tên thuộc họ La tinh hoặc đã phiên âm ra La tinh. 


\section{MA TRẬN SAI SÓT VÀ PHÂN LÓP KÝ TỰ}

Trong [3], [4] đã trình bày một thuật toán để truy nguyên đồng nhất tên họ, gọi là thuật toán truy nguyên codfonetics (truy nguyên theo mã phát âm). Thuật toán này còn khá nhiều khiếm khuyết khi sử dụng ở Việt Nam. Để khắc phục những hạn chế của thuật toán, đã tiển hành các quan sát phân tích trên khoảng 25 ngàn cặp mẫu tên họ, đã lập được ma trận độ đo sai sót (gọi tắt là ma trận sai sót) biểu thị tần suất sai lệch giữa các kí tự trong bảng chữ cái $A \rightarrow Z$.

Có thể liệt kê một số nguyên nhân chính dẫn đến sai lệch giữa các kí tự đã nêu như sau:

- Viết tên họ theo phát âm.

- Ngôn ngữ khác nhau dẫn đến cách viết khác nhau.

- Yếu tố tâm lý, khi mệt mỏi thường viết lẫn đặc biệt ở cuối từ.

- Gõ nhầm trên bàn phím.

- Sai số trên đường truyền.

Gọi $P\left(X_{i}, X_{j}\right), 1 \leq i, j \leq 26$ là độ đo sai sót của kí tự ở vị trí thứ $i$ sang kí tự đơ vị trí thứ $j$ trong bảng chữ cái Latin từ $A$ đến $Z, P\left(X_{i}, X_{j}\right)$ chính là một phần tứ trong ma trận độ đo sai sót. Xác định hàm $R\left(X_{i}, X_{j}\right)$ như sau:

$$
R\left(X_{i}, X_{j}=1-\frac{P\left(X_{i}, X_{j}\right)+P\left(X_{j}, X_{i}\right)}{2}\right.
$$

Hàm $K\left(X_{i}, X_{j}\right)$ đo độ "gần" giữa $X_{i}$ và $X_{j}$ xác định như sau:

$$
K\left(X_{i}, X_{j}\right)=\frac{1}{1+a^{*} R\left(X_{i}, X_{j}\right)^{2}}=\frac{1}{1+\alpha\left(1-\frac{P\left(X_{i}, X_{j}\right)+P\left(X_{j}, X_{i}\right)}{2}\right)^{2}}
$$

Ở đây $\alpha=\frac{1}{2^{5}}$.

Trong [3] đã sử dụng thuật toán phân loại tự động với các công thức (2.1), (2.2) để phân loại bảng chữ cái trong vùng tên họ thành các nhóm, mỗi kí tự trong nhóm có độ sai lệch trung bình sang các kí tự cùng nhóm là lớn nhất so với các kí tự của nhóm khác.

Thực tế kiểm định, phân bảng chữ cái $A \rightarrow Z$ thành 8 nhóm với các đại diện sau đây là tối ưu:

Nhóm 1 là $A$

Nhóm 2 là $M$

Nhóm 3 là $N$

Nhóm 4 là $W$
Nhóm 5 là $V$

Nhóm 6 là $I$

Nhóm 7 là $Z$

Nhóm 8 là $B$ 
Các nhóm nói trên được sử dụng để tạo codfonetics cho các tù̀ trong thuộc tính tên họ.

Sai sót theo vị trí và việc chọn ngưỡng

Các kí tự ở từng vị trí khác nhau trong từ, tần suất sai sót cũng khác nhau. Thống kê trên 2 ngàn cặp tên có sai sót, lập bảng tần suất sai sót theo vị trí; ở các vị trí $1-3$, độ sai lệch dao động ở khoảng $5,1 \%$, sai nhiều nhất ở các vị trí thứ 5 và 6 sai lệch tới $55 \%$.

\section{XÁC ĐỊNH NGUOÕNG Độ ĐO}

$X$ được coi là trùng với $Y$ nếu:

- 3 kí tự đầu (của $X$ và $Y$ ) sai khác nhau không quá 1 kí tự

- Sau kí tự thứ 3 , sai khác nhau không vượt quá 2 trong mối 5 kí tự

Kí hiệu $s_{1}$ là tống số 3 kí tự (đầu khác trắng), $\delta_{1}$ là số kí tự sai sót trong 3 kí tự đầu, $s_{2}$ là tổng số kí tự sau kí tự thứ 3 (khác trắng), $\delta_{2}$ là số kí tự sai trong số xác kí tự thứ 3 . Khi đó có thể viết:

$$
\delta_{1}=\sum_{i=1}^{3} \rho\left(X_{i}, Y_{i}\right), \quad \delta_{2}=\sum_{i=4}^{n} \rho\left(X_{i}, Y_{i}\right)
$$

và do đó

$$
\rho(X, Y)=\sum_{i=1}^{3} \rho\left(X_{i}, Y_{i}\right)+\sum_{i=4}^{n} \rho\left(X_{i}, Y_{i}\right)=\delta_{1}+\delta_{2}
$$

Hiền nhiên rằng từ 3.1 và 3.2 suy $\mathrm{ra} X \equiv Y$ nếu $\delta_{1} \leq \frac{1}{3} s_{1}$ và $\delta_{2} \leq \frac{2}{5} s_{2}$.

Vấn đề còn lại là tìm giải thuật so sánh hai từ $X$ và $Y$

\section{THUẬT TOÁN}

Gọi tam giác lân cận của kí tự bất kỳ trong hai từ

$$
X=\left\{X_{1} X_{2} X_{3} \ldots X_{n}\right\}, \quad Y=\left\{Y_{1} Y_{2} Y_{3} \cdots Y_{m}\right\}, \quad n, m \leq 24
$$

là kí tự của tù này, aược xem xét với $\$$ kí tụ trước một, cùng và sau một kí tự của kí tư trong tù kia.

Ví dụ, tam giác lân cận của $X_{i}$ là $\left(Y_{i-1}, Y_{i}, Y_{i+1}\right)$. 


\section{Quy tắc so sánh hai từ được xác lập như sau:}

Tiến hành so sánh từng kí tự giữa hai từ.

- Kí tự thuộc từ này, so sánh với kí tự ở từ kia trong phạm vi tam giác lân cận, gọi là giải thuật so sánh chéo nhau, trước hết là so sánh kí tự ở vị trí thẳng hàng.

- Trường hợp các từ độ dài khác nhau thì so sánh lân cận bắt đầu từ vị trí duôi các từ ngược trở lại đầu từ, gọi là giải thuật so sánh giất lùi.

Gọi $e_{1}$ là số kí tự sai trong 3 kí tự đầu, $s_{1}$ là tồng số số kí tự (khác trắng) trong 3 kí tự đầu, $e_{2}$ là số kí tự từ kí tự thứ $4, s_{2}$ là tông số số kí tự (khác trắng) từ kí tự thứ 4.

Nếu $X \equiv Y$ thì có các biểu diễn sau:

- Với 3 kí tự đầu $\delta_{1} \leq \frac{1}{3} s_{1}$, khi đó $e_{1} \leq 1$ nếu $s_{1}=3$

$$
e_{1}=0 \text { nếu } s_{1} \leq 2
$$

- Các kí tự sau $\delta_{2} \leq \frac{2}{5} s_{2}$, khi đó $e_{2} \leq 1$ nếu $3 \leq s_{2} \leq 4$

$$
e_{2} \leq 2 \text { nếu } 5 \leq s_{2} \leq 7
$$

(trường hợp tham khảo: $e_{2} \leq 1$ nếu $e_{1}=0$ và $0<s_{2} \leq 2$ )

Thuật toán so sánh hai từ như sau:

Trường hợp 1: $X$ thuộc trọn trong $Y(X \subset Y)$

Không giảm tổng quát coi như $X$ trùng với $Y$ ơ đoạn đầu, từ trái sang sang phải.

$e_{1}+e_{2}=(m-n)$ và sử dụng quy tắc (4.1) và (4.2) để quyết định xem $X=Y$ hay không?

Trường hợp thứ hai: $X$ và $Y$ bất kỳ

- Nếu $n=m$ (hai từ có độ bằng nhau), thực hiện giải thuật tìm cặp chéo nhau.

- Nếu $n \neq m$ (hai từ có độ dài bằng nhau), thực hiện giải thuật so sánh giật lùi.

GIẢI THUẬT tìm cặp chéo nhau

Bước 1. Ba kí tự đầu tính $\sum_{i=1}^{3} \rho(X i, Y i)$

So troơng úng $X_{i}$ với $Y_{i}$,

nếu $X_{i} \neq Y_{i}$, tăng $e_{1}$ lên $1\left(e_{1}=e_{1}+1\right)$, tăng $i$ lên $1(i=i+1)$

Với mỗi $i$, kiểm tra $e_{1}$

Nểu $e_{1} \geq 2$ thì hai từ khác nhau, kết thúc so sánh 
VỀ GIẢI THUẬT SO SÁNH HAI TỪ TRONG THUẬT TOÁN TRUY NGUYÊN ĐỒNG NHẤT 95

Nếu $i>3$, chuyển tới buớc 2

Bước 2. Các kí tự sau kí tự thế 3 , tính $\sum_{i=4}^{n} \rho(X i, Y i)$

Thực hiện giải thuật tim cặp kí tụ chéo nhau

Bước 3. So sánh từng kí tự $X_{i}$ với $Y_{i}$

Nếu $X \equiv Y$, tăng $i$ lên $1(i=i+1)$, lặp lại bước 3

Ngược lại $\left(X_{i} \neq Y_{i}\right)$, tăng $e_{2}$ lên $1\left(e_{2}=e_{2}+1\right)$ chuyến tới bước 4

Bước 4. So sánh $X_{i+1}$ ví $Y_{i}$

Nếu $X_{i+1} \equiv Y_{i}$, có nưa dường chéo trên $\left(X_{i+1}, Y_{i}\right)$

Ghi nhận vị trí $Y_{i}$

So sánh $X_{i}$ với $Y_{i+1}$

Nếu $X_{i} \equiv Y_{i+1}$, có nứa đường chéo dưới $\left(X_{i}, Y_{i+1}\right)$

Ghi nhận vị trí $X_{i}$

Ghi nhận có cắp chéo cặp, không tính sai $\left(e_{2}=e_{2}-1\right)$

Tăng $i$ lên $2(i=i+2)$

Lặp lại buớc 3

Nếu $X_{i} \neq Y_{i+1}, X_{i}$ là kí tự thừa (chèn), coi như sai

Tăng $i$ lên $1(i=i+1)$

Lặp lại bước 9

Nếu $X_{i+1} \neq Y_{i}$, so sánh $X_{i}$ với $Y_{i+1}$

Nếu $X_{i} \equiv Y_{i+1}$, có nứa đường chéo trên $\left(X_{i}, Y_{i+1}\right)$

$Y_{i}$ là kí tự thứa (chèn)

Ghi nhận vị trí $X_{i}$

Tăng $i$ lên $1(i=i+1)$

Lặp lại bước 2

Nếu $X_{i} \neq Y_{i+1}$, có hai cặp đều sai là $\left(X_{i}, Y_{i}\right)$ và $\left(X_{i+1}, Y_{i+1}\right)$

tăng $e_{2}$ lên $2\left(e_{2}=e_{2}+2\right)$

tăng $i$ lên $2(i=i+2)$

Lặp lại buớc $\$$

Trong quá trinh tính toán, $e_{2}$ luôn a̛ược kiềm tra với nguõ̃ng, nếu vuợt quá ngướng ở bất cứ buớc nào, đều dùng, ngược lai, kết hợp với $e_{1}$ dể quyết định xem $X \equiv Y$ hay không.

GIẢI THUẬT so sánh giật lùi

Nếu $n \neq m$ (hai tù dộ dài không băng nhau), ta chí xét các tù hơn kém nhau không vuợt quá 2 kí tự. 
Không mất tính tồng quát, giả sử $m=n-1$, tức là $X$ dài hơn $Y$ một kí tự (chính là $X_{n}$ ).

Bước 1. Ba kí tự đầu, tính $\sum_{i=1}^{3} \rho\left(X_{i}, Y_{i}\right)$

So tương ứng $X_{i}$ với $Y_{i}$ như bước 1 thuật toán tìm cặp chéo

Bước 2. Các kí tự sau kí tự thứ 3 , tính $\sum_{i=4}^{n} \rho\left(X_{i}, Y_{i}\right)$

Thực hiện giải thuật toán so sánh "giật lùi",

So sánh $X_{n}$ với $Y_{m}$

Nếu $X_{n}=Y_{m}$ thì $X_{n}$ khôg phải là kí tự thừa

Bỏ cặp kí tự $\left(X_{n}, Y_{m}\right)$, thay $Y_{m}$ bằng dấu *

Quay lại trường hợp so sánh 2 chuỗi bằng nhau

$\left(X_{1}, X_{2}, \ldots X_{m}\right)$ với $\left(Y_{1}, Y_{2}, \ldots Y_{m}\right)$, với $Y_{m}=*$

Nếu $X_{n} \neq Y_{m}$ thì $X_{n}$ là kí tự thừa, bỏ $X_{n}$, gán $e_{2}=1$

Quay lại trường hợp so sánh 2 chuỗi bằng nhau

$\left(X_{1}, X_{2}, \ldots X_{m}\right)$ với $\left(Y_{1}, Y_{2}, \ldots Y_{m}\right)$

\section{5. ĐÁNH GIÁ THUẬT TOÁN}

- Khắc phục về cơ bản các hạn chế của thuật toán No. 1 trong [3], nhất là đã tạo codfonetics đủ mịn, phản ánh đúng thực tế quy luật sao sót ngẫu nhiên; mặt khác tận dụng các trường hợp không đủ thông tin nhưng vẫn tìm kiếm để giúp việc xác minh được thực hiện trên các tập hạn chế về số lượng.

- Tốc độ tìm kiếm rất nhanh đối với trường hợp tên họ có độ dài bằng nhau.

- Đã đồng nhất hàng triệu trường hợp, độ chính xác cao.

- Tập hợp được hàng chục ngàn trượng hợp tên họ, năm sinh có sai sót và với số lượng mẫu đồng nhất này, ma trận sai sót không ngừng được hoàn chinh và thuật toán liên tục được bổ sung (tự học).

- Thuật toán trên được triển khai cài đặt trong hệ thống thông tin Bộ Nội vụ đạt hiệu quả tốt. Hoàn toàn có giá trị ứng dụng trong các hệ thống thông tin tương tự.

\section{TÀI LIỆU THAM KHẢO}

1. Phương Minh Nam, Phân tích hệ thống và phần mềm hệ thống thông tin $X N C$, Báo cáo tổng kết đề tài NCKH cấp nhà nước, mã số 90-68-051. 
2. Phương Minh Nam, Đồng nhẩt trong cơ sở dĩ liệu xuẩt nhập cảnh, Tạp chí NCKHCA, T. 3 (1997) 38-41.

3. Phương Minh Nam, Đồng nhất trong co sớ dữ liệu tên họ Latin, Tạp chí Tin học và Điều khiển học, T. 12, S. 2 (1997) 31-41.

4. Nguyễn Doãn Tiến, Trương Đức Thái, Phương Minh Nam, Về thuật toán đồng nhất theo trọng số, Ki yểu Tuần lễ tin học lần thứ $2-1992$, tại Tp. Hồ Chí Minh.

(1) Cuc Quán lý XNC Bộ Nội vu.

(2) Viện Diều tra quy hoạch đất đải.

Nhận bài ngày 12-7-1997 